\title{
Robust Low-Power Algorithm for Random Sensing Matrix for Wireless ECG Systems Based on Low Sampling-Rate Approach
}

\author{
Mohammadreza Balouchestani*, Kaamran Raahemifar, Sridhar krishnan \\ Electrical and Computer Engineering Department, Ryerson University, Toronto, Canada. \\ Email: mbalouch@ee.ryerson.ca, kraahemi@ee.ryerson.ca, Krishna@ee.ryerson.ca
}

Received April, 2013.

\begin{abstract}
The main drawback of current ECG systems is the location-specific nature of the systems due to the use of fixed/wired applications. That is why there is a critical need to improve the current ECG systems to achieve extended patient's mobility and to cover security handling. With this in mind, Compressed Sensing (CS) procedure and the collaboration of Sensing Matrix Selection (SMS) approach are used to provide a robust ultra-low-power approach for normal and abnormal ECG signals. Our simulation results based on two proposed algorithms illustrate $25 \%$ decrease in sampling-rate and a good level of quality for the degree of incoherence between the random measurement and sparsity matrices. The simulation results also confirm that the Binary Toeplitz Matrix (BTM) provides the best compression performance with the highest energy efficiency for random sensing matrix.
\end{abstract}

Keywords: Sensing Matrix; Power Consumption; Normal and Abnormal ECG Signal; Compressed Sensing; Block Sparse Bayesian learning

\section{Introduction}

WBANs as a special purpose of Wireless Sensor Networks (WSNs) consist of tiny Biomedical Wireless Sensors (BWSs) and a Gate Way (GW) to connect to the external databases in the hospital and medical centers [1]. The WBANs are expected to be a breakthrough in healthcare areas such as hospital and home care, Mobile Health (MH), Electronic Health (EH), and physical rehabilitation. The GW could connect the BWSs, to a range of wireless telecommunication networks. These wireless telecommunication networks could be either mobile phone networks, standard telephone networks, dedicated medical center or using public Wireless Local Area Networks (WLANs) nodes also known a Wi-Fi system [2]. The compressed sensing is a revolutionary idea for the acquisition and recovery of sparse signals that enables sampling-rate significantly below the classical Nyquist-rate (NR). The electrocardiogram (ECG) signals are widely used in health care systems because they are noninvasive mechanisms to establish medical diagnosis of heart diseases. The current ECG systems suffer from important limitations: limited patient's mobility, limited energy, limited on wireless applications. In order to fully exploit the benefits of WBANs such as $\mathrm{EH}, \mathrm{MH}$, and Ambulatory Health Monitoring Systems (AHMS) the power consumption and sampling rate should be restricted to minimum. Long-term records of ECG signals in WBANs have become commonly used to collect information from the heart for diagnostic and therapeutic purposes [3]. That is why the quantity of data grows significantly and compression is required for reducing the storage, transmission times, and power consumption. The ECG signals generally illustrate the redundancy between adjacent heartbeats due to its semi-periodic structure [4]. It is evident that this redundancy provides a high fraction of common support between consecutive heartbeats that is a good candidate for compression. However, they have low-frequency and non-stationary features and processing noise setting strong characters, neither time-demine nor frequency-domain based methods are suitable for analyzing these signals. This paper presents new algorithms with contribution of CS approach in mind, and SMS procedure based on Dynamic Thresholding Approach (DTA) to establish a robust ultra-low-power for normal and abnormal ECG signals. The CS theory indicate that $\mathrm{s}$ a small number of random linear measurements of sparse signals contain enough information to collect, process, transmit, and recover the original signal [5]. This approach emphasizes that the signal representing sparsity in any orthogonal basis can 
be well reconstructed using $\ell_{1}$ norm minimization, while satisfying the Restricted Isomerty Property (RIP) condition for random measurement matrix $\Phi$ and orthogonal $\Psi$ in any domain [6]. Our simulation results based on two proposed algorithms illustrate $25 \%$ decrease in sampling- rate and a good level of quality for the degree of incoherence between the random measurement and sparsity matrices. The simulation results also confirm that the Binary Toeplitz Matrix (BTM) provides the best compression performance with the highest energy efficiency for random sensing matrix. The structure of this paper is organized as follows: Section 2 gives an overview about CS theory in general and specifically for WBANs. Section 3 proposes the new algorithm based on combination of CS theory, and SMS approach. The reminder of the paper is categorized in the following way: the simulation results are presented in Section 4. The conclusion is drawn in Section 5.

\section{Overview of Compressed Sensing}

The conventional sampling approaches have traditionally relied on the Shannon sampling theorem. This theory says a signal must be sampled at least twice its bandwidth in order to be represented without error. The traditional approaches have two important drawbacks. First, they generate huge samples for many applications with large bandwidth that is not tolerated. Second, even for low signal bandwidths such as ECG signals, they produced a large amount of redundant digital samples. That is why it is desirable to reduce the number of acquired ECG samples by using advantages of the sparsity. The CS theory replaces the conventional sampling and reconstruction operation with a general random linear measurement process and an optimization scheme in order to recover original signal from a small number of random measurements.

\subsection{Basic Theorem}

The goal in the digital-CS theory as a new sampling scheme is to reduce the load of sampling-rate by decreasing the number of samples after the Analog to Digital Convertor (ADC) required to completely describe a signal by exploiting its compressibility [7]. An important aspect of CS theory is that the measurements are not point samples but more general linear functions of the signals. Any compressible or sparse signal $\mathbb{D}$ in $\mathbb{R}^{\mathrm{N}}$ can be expressed as:

$$
D=\sum_{i=1}^{N} C_{i} \Psi_{i}
$$

The compresses signal $\mathbb{C}$ can also be found as:

$$
[\mathbb{C}]_{M \times 1}=[\Phi]_{M \times N}[D]_{N \times 1} .
$$

Thus, the compressed signal is found as:

$$
[\mathbb{C}]_{M \times 1}=[\Phi]_{M \times N}[\Psi]_{N \times N}[C]_{N \times 1}=[\Theta]_{M \times N}[C]_{N \times 1} \text {. }
$$

Fortunately, $[\Phi]$ and $[\Theta]$ have two interesting and useful properties. First, they are incoherent with the basis $[\Psi]$. Second, they have the RIP with high probability where is suitable condition to recover the original signal in the receiver side [8]. Thus, CS scenario has two important steps. First step in CS offers a stable measurement matrix $[\Phi]_{M \times N}$ to ensure that the salient information in any compressible signal is not damaged by the dimensionality reduction from $D \in \mathbb{R}^{\mathrm{N}}$ down to $\mathbb{C} \in \mathbb{R}^{\mathrm{M}}$. In the second step, the CS theory offers a reconstruction algorithm under certain condition and enough accuracy to recover original signal $D$ from the compressed signal. Therefore, we can exactly reconstruct the original signal $D$ with high probability via $\ell_{1}$ norm by solving the following convex optimization problem $\left(\|s\|_{1}=\sum_{n}\left|s_{n}\right|\right)$ :

$$
\begin{aligned}
& \min \|s\|_{1} \text { subject to } \mathbb{C}=\Phi \Psi S \text {. } \\
& s \in \mathbb{R}^{N}
\end{aligned}
$$

There are two important conditions, which guarantee the correctness of this recovery. Firstly, the number of random linear measurements, the number of coefficients, and the number of non-zero coefficients must satisfy the following equation [9]:

$$
M \leq K / C(\log N) .
$$

Secondly, for any vector $a$ of the original signal $[D]$ matrix $[\Phi]$ must satisfy the following condition for some $\varepsilon \succ 0$ :

$$
1-\varepsilon \leq\|\Phi a\|_{2} /\|a\|_{2} \leq 1+\varepsilon
$$

where satisfies RIP property for the random dictionary matrix. In order to recover $K$-sparsity of the original signal, now we have $M \times K$ system of linear equations, with $M$ equations and $K$ unknowns. It is possible to find out the $K$-sparsity of the original signal, because of $M \geq K$.

\subsection{Compressed Sensing in WBANs}

The CS theory says sparse or compressible signals such as ECG; signals can be well recovered using to minimize $\ell_{1}$ norm optimization, while satisfying the RIP condition for the random measurement matrix $\Phi$ and orthogonal basis $\psi$. Basically, the biomedical signals are sparse or near sparse. To verify this condition, we exploit a conventional Fast Fourier transformation (FFT) to check signal sparsity. These signals have $K$ non-zero coefficients and $(N-K)$ zero coefficients with $K \ll N$ and can be well recovered using $M$ projects or measurements 
such as $K \leq M<<N$. As the result, the small number of non-zero coefficients is small; the CS theory can be applied to reduce the load of sampling. Figure 1 illustrates CS theory in WBANs.

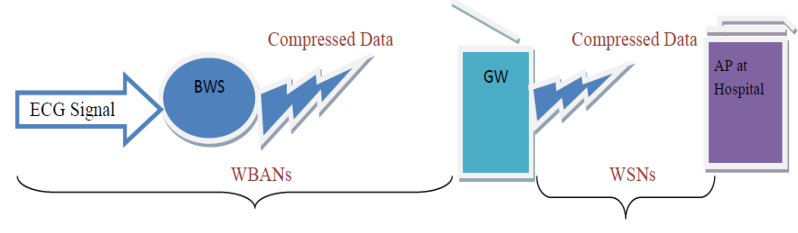

Figure 1. CS in WBANs.

As it can be seen the biomedical signals are compressed by wireless sensors. The collected compressed biomedical data are then transmitted wirelessly to Access Points (APs) at hospital, ambulance, and helicopter [10, 11]. The APs recover compressed biomedical data for diagnostic and therapeutic purposes. Furthermore, the $D$ data vector in WBANs is a sparse vector, because the GW needs to collect only $M$ bits instead of $N$ bits of data ( $M \approx K-$ sparse) through the network. In the WBANs with $N$ wireless sensor, sensor $i$ is acquiring a sample $d_{i}$ of the human body [9]. The final goal in WBANs for medical applications is to collect Data's vector $D$ of $N$ wireless sensors in a suitable basis $\Psi=\left[\Psi_{1}\right]\left[\Psi_{2}\right] \ldots\left[\Psi_{\mathrm{N}}\right]$ like:

$$
D=\sum_{i=1}^{N} d_{i} \Psi_{i}
$$

CS suggests that, under certain conditions, instead of collecting data vector $D$, we can collect compressed vector $[\mathbb{C}]=[\Phi][D]$ where $\Phi$ is $(K \times N)$ sensing matrix whose entries are i.i.d random variables. In non-CS scenario a node is receiving $N-1$ packets and sends out $N$ packets $((N-1)$ received packets plus its data) each packet corresponding to data sample from a node. In WBANs with CS theory the GW needs only to receive $M$ ( $M \approx K$-sparse) packets [9]. In order to use CS, each node needs to know the value of Compressed Ratio $(C R=N / K)$ that is constant and value of $N$ [16]. The node $i$ compute $K=N / C R$ and generate $K$ values $\Phi_{j i}(1 \leq j \leq k)$ and creates a vector $D_{i}\left[\Phi_{1 i}, \Phi_{2 i \ldots} \Phi_{k i}\right]$, where $D_{i}$ is its own data. Typically, node $i$ would wait to receive from all its downstream neighbors. Each received packet carries its index from 1 to $\mathrm{K}$, so that it can be added to the data already waiting in $i$ with the same index (either locally produced or received from a neighbor). Then node $i$ would send exactly $\mathrm{K}$-Packets corresponding to the aggregated column vectors. Now the difference between CS and non-CS operation becomes clear [10]: CS operation requires each node to send exactly $M$ packets irrespective of what it has received, and each node needs to know $C R$ and $N$ and then computes the value of $(M \approx K)$. The received vector in $\mathrm{GW}$ can be written as:

$$
[\mathbb{C}]_{M \times 1}=[\Phi]_{M \times N}[D]_{N \times 1} .
$$

Consequently, the received vector in GW is a condensed representation of the sparse events and can be expressed like:

$$
\left(\begin{array}{c}
\mathbb{C}_{1} \\
\vdots \\
\mathbb{C}_{M}
\end{array}\right)=\left(\begin{array}{ccc}
\Phi_{11} & \cdots & \Phi_{1 N} \\
\vdots & \vdots & \vdots \\
\Phi_{M 1} & \cdots & \Phi_{M N}
\end{array}\right)\left(\begin{array}{c}
D_{1} \\
\vdots \\
D_{N}
\end{array}\right) .
$$

Our simulation results show that by employing the CS the WBANs can achieve a higher transmission, a lower time delay and higher probability of success of data transmission. Therefore, a combination of CS theory to WBANs is an optimal solution for achieving robust WBAN with low sampling rate and power consumption. As it can be seen the biomedical signals are compressed by wireless sensors. The collected compressed biomedical data are then transmitted wirelessly to Access Points (APs) at hospital, ambulance, and helicopter [10, 11]. The APs recover compressed biomedical data for diagnostic and therapeutic purposes.

\section{Proposed Approach}

To validate the performance of the considered compression schemes three performance measurements are defined in this section first. Then, the proposed algorithm for selecting the best fit for random sensing matrix is proposed.

\subsection{Performance Measure}

The Compression Ratio (CR), the Structural Similarity Index (SSI), and Percentage Root-mean-square Difference (PRD) are employed as performance measures in our approach. The CR is found as follows [12]:

$$
C R=N / M \times 100,
$$

where $M$ and $N$ are the number of random linear measurements and number of samples in ECG signals respectively. Further, our simulation results indicate that satisfying quality of $S R$ can be achieved when $C R$ does not exceed of 35\%. The SSI metric is defined as [13]:

$$
S S I=(\mathbb{C} / D) \times 100,
$$

where $D$ and $\mathbb{C}$ are the original and recovered ECG signals respectively. This metric measure the similarity between the recovered and original ECG signals [14]. Higher SSI means better recovery quality. Our simulation results will show the proposed approach has this ability to achieve SSI with value close to $100 \%$. The PRD is computed as [14]: 


$$
P R D=\left(\|D-\mathbb{C}\|_{2} /\|D\|_{2}\right) \times 100 .
$$

The value of $P R D$ shows the quality of reconstruction approach. The relationship between the measured $P R D$ and diagnostic distortion is recognized on the weighted diagnostic data for ECG signals, which classifies the different values of $P R D$ based on the signal quality obtained by a specialist. Table 1 illustrates the resulting different quality classes and corresponding $P R D$ values. As depicted in Table 1, lower $P R D$ means better recovery quality.

Table 1. Different Quality Classes.

\begin{tabular}{ll}
\hline$P R D$ & Quality of recovery \\
$0 \sim 1 \%$ & Excellent \\
$1 \sim 2 \%$ & Very good \\
$2 \sim 0.85 \%$ & Good \\
$\geq 0.85 \%$ & Poor \\
\hline
\end{tabular}

\subsection{Proposed SMS Algorithm}

The random measurement matrix $[\Phi]$ is a key component of CS theory. Two key features are needed for a successful implementation of CS approach: Sparsity of the biomedical signal and incoherence between the random sensing matrix and the sparsity basis [15]. That is why; the random sensing matrix must exhibit a high degree of incoherence with the sparsity basis $[\Psi]$. In this part, the new SMS procedure is presented to select the best fit for the random sensing matrix $[\Phi]$. Herein, Bernoulli Toeplitz, Gaussian Circulant, and Binary Toeplitz matrices are examined to find out the best fit for random sensing matrix [16]. The Toeplitz matrix is a matrix in which each descending diagonal from left to right is constant. The random sensing matrix in Binary form is expressed as:

$$
\Phi=\left(\begin{array}{cccc}
\Phi_{0} & \Phi_{-1} & \cdots & \Phi_{-n+1} \\
\Phi_{1} & \Phi_{0} & \cdots & \Phi_{-n} \\
\vdots & \vdots & \Phi_{0} & \vdots \\
\Phi_{n-1} & \cdots & \Phi_{1} & \Phi_{0}
\end{array}\right)
$$

The Circulant matrix is a special kind of Toepliz matrix where each row vector is rotated on element to the right relative to the preceding row vector [17]. The random sensing matrix in Circulant form is illustrated as:

$$
\Phi=\left(\begin{array}{cccc}
\Phi_{0} & \Phi_{n-1} & \cdots & \Phi_{1} \\
\Phi_{1} & \Phi_{0} & \cdots & \Phi_{2} \\
\vdots & \vdots & \Phi_{0} & \vdots \\
\Phi_{n-1} & \cdots & \Phi_{1} & \Phi_{0}
\end{array}\right)
$$

In the simulation part, CS approach is applied on the ECG data obtained from MIT-BIH database for three sensing matrix possibilities: (1) Bernoulli Toeplitz matrix, (2) Gaussion Circulant matrix, and (3) Binary Toeplitz matrix. Our simulation results will further confirm that the Binary Toeplitz matrix shows the best performance for the random sensing matrix $\Phi$. Table 2 illustrates our new algorithm to select the best fit for random sensing matrix $\Phi$.

Table 2 . The best fit for sensing matrix.

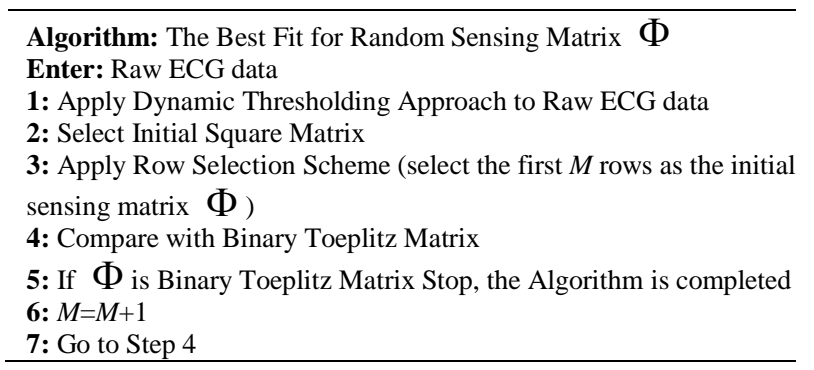

In the step 1 of the proposed algorithm, the DTA procedure is applied to the raw ECG data. The principal objective of the DTA is to vary the sparsity level of a raw ECG signal to convenient level [18]. In the simulation part, the convenient level is defined $98 \%$. In the step 2 , the initial square matrix is used for each of the sensing matrices in the experiments [19]. In the step 3, a Row Selection Scheme (RSS) is applied to reduce the number of rows from $N$ to $M$ [20]. Two RSSs approaches are compared: (1) select first $M$ rows from the initial $N \times N$ matrix, and (2) randomly select $\mathrm{M}$ rows from the initial $N \times N$ matrix. The first RSS approach demonstrates better performance than the second RSS approach. So only the first RSS approach is utilized in the proposed algorithm.

\section{Simulation Results}

The following assumptions were made for simulation:

- Experiments are carried out over a 10-minutes ECG signal from MIT-BIH database [21].

- One hundred repletion's are averaged for our simulation results. To validate the simulation results ECG signals from records 100,107,115 and 117 of MIT-BIH are investigated.

- The mean of ECG blocks is rounded in the sliding window to the nearest multiple of $2^{L}$, where $L$ is the BSBL level [22].

- To simulate SNR for ECG signals the following equation is used [23].

$$
S N R=-20 \log _{10}(0.01 P R D) .
$$

Three sensing matrix possibilities are examined for random sensing matrix $\Phi$ : (1) Bernoulli Toeplitz matrix, (2) Gaussian Circulant matrix, and (3) Binary Toeplitz 
matrix [24].

- The SPARCO toolbox is used for testing sparse reconstruction algorithm.

- The SPGL1 (Spectral Projected Gradient for $\ell_{1}$ minimization) toolbox is used to determine Large-scale one-norm regularized least squares in the following equation:

$$
\begin{aligned}
& \min \|c\|_{1} \text { subject to } \mathbb{C}=\Phi D . \\
& c \in \mathbb{R}^{N} .
\end{aligned}
$$

To validate the simulation results, the BPBQ (Basis Pursuit DeQuantizer) toolbox is used for recovery of sparse signals from quantized random measurements to solve [25]:

$$
\underset{c \in \mathbb{R}^{N}}{\arg \min \|c\|_{1} \text { subject to }\|\mathbb{C}-\Phi D\|_{p} \text { for } p \geq 2 .}
$$

The simulation results were obtained for an input signal of $N=512$ samples and a 12-bits resolution for the input signal and the measurement signal $\mathbb{C}$.

- To simulate the SMS approach, the DTA framework is used to vary the sparsity level [26].

Figure 2 illustrates the sampling-rate for random binary matrix with CS theory for different values of non-zero entries $K$ for specific records of ECG signals.

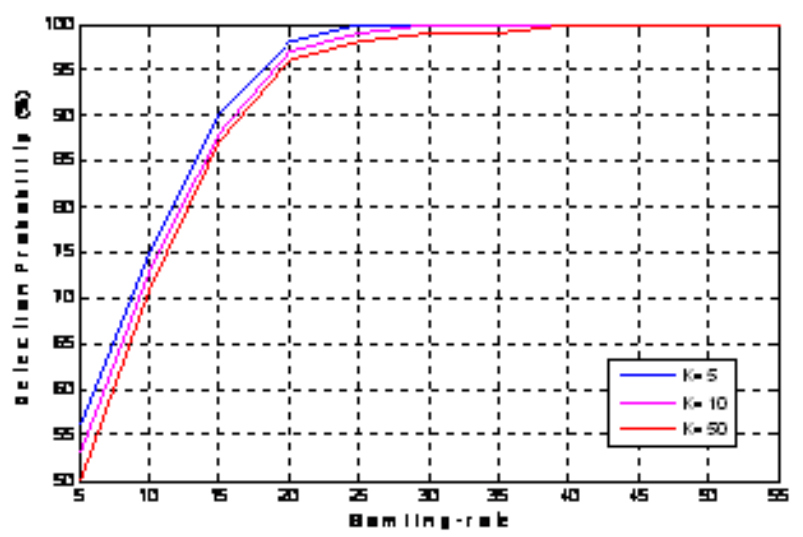

Figure 2. Sampling-rate.

Based on the results of Figure 2 and suitability of the random binary matrix, the sampling rate can be reduced by $75 \%$ of NR without sacrificing the performance. Figure 3 shows simulation results on power consumption for random binary matrix with CS theory in terms of Compressed Ratio (CR) for specific records of ECG signals.

As depicted in the Fig. 3, the power consumption can be reduced by $65 \%$ by employing CS theory. Table 3 compares the simulation results on sampling rate and power consumption for random binary matrix with CS theory.

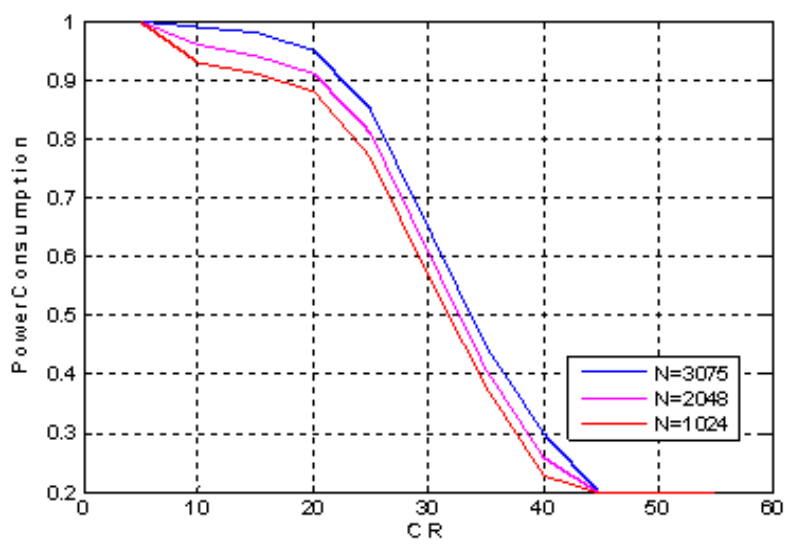

Figure 3. The power consumption.

Table 3. Comparing SR and PC.

\begin{tabular}{cccc}
\hline $\boldsymbol{N}$ in ECG & CR & SR & PC \\
\hline $\mathbf{1 0 2 4}$ & 10.24 & $25 \% *(\mathrm{NR})$ & $30 \% *($ PC in non-CS $)$ \\
$\mathbf{2 0 4 8}$ & 20.48 & $28 \% *(\mathrm{NR})$ & $35 \% *(\mathrm{PC}$ in non-CS $)$ \\
$\mathbf{3 0 7 4}$ & 30.74 & $32 \% *(\mathrm{NR})$ & $40 \% *($ PC in non-CS $)$ \\
\hline
\end{tabular}

Table 2 indicates that satisfying quality on sampling rate and power consumption can be achieved when CR does not exceed of 30 .

\section{Future Work}

We have simulated the benefit of CS to wireless ECG systems for some recodes of ECG signals. Our future work involves developing the CS theory to other records of ECG signal, including abnormal records for wireless ECG systems.

\section{Conclusions}

The ECG signal is widely used in WBANs because it is a noninvasive way to provide medical diagnosis of heart diseases. This paper has presented new algorithm with a contribution of CS approach, and SMS procedure based on DTA aapproach to establish a robust ultra-low-power for normal and abnormal ECG signals. The works by Alvarado [2] and Baheti [14] focus only CS theory for normal ECG signal with only random Gaussian matrix. While the present study offers a new algorithm to select the best fit for random sensing matrix of the CS approach for normal and abnormal ECG signals. Our simulation results validate the suitability of a new algorithm for a real-time energy-efficient ECG compression on resource-constrained in WBANs. The simulation results also confirm that the Binary Toeplitz matrix provides the best compression performance with the highest energy efficiency for random sensing matrix. Advanced ECG 
systems based on CS will be able to deliver healthcare not only to patients in hospital; but also in their homes.

\section{REFERENCES}

[1] M. Balouchestani, K. Raahemifar and S. Krishnan, "Low Power Wireless Body Area Networks with Compressed Sensing Theory," in Circuits and Systems (MWSCAS), 55th IEEE International Midwest Symposium on, 2012, pp. 916-919.

[2] A. S. Alvarado, C. Lakshminarayan and J. C. Principe, "Time-Based Compression and Classification of Heartbeats," Biomedical Engineering, IEEE Transactions on, Vol. 59, 2012, pp. 1641-1648. doi:10.1109/TBME.2012.2191407

[3] A. M. R. Dixon, E. G. Allstot, D. Gangopadhyay and D. J. Allstot, "Compressed Sensing System Considerations for ECG and EMG Wireless Biosensors," Biomedical Circuits and Systems, IEEE Transactions on, Vol. 6, pp. 156-166, 2012. doi:10.1109/TBCAS.2012.2193668

[4] B. H. Tracey and E. L. Miller, "Nonlocal Means Denoising of ECG Signals," Biomedical Engineering, IEEE Transactions on, Vol. 59, No. 9, 2012, pp. 2383-2386. doi:10.1109/TBME.2012.2208964

[5] H. Sjoland, J. B. Anderson, C. Bryant, R. Chandra, O. Edfors, A. J. Johansson, N. S. Mazloum, R. Meraji, P. Nilsson, D. Radjen, J. N. Rodrigues, S. M. Y. Sherazi and V. Owall, "A Receiver Architecture for Devices in Wireless Body Area Networks,” Emerging and Selected Topics in Circuits and Systems, IEEE Journal on, Vol. 2, 2012, pp. 82-95. doi:10.1109/JETCAS.2012.2186681

[6] Y. Hamada, K. Takizawa and T. Ikegami, "Highly Reliable Wireless Body Area Network Using Error Correcting Codes," in Radio and Wireless Symposium (RWS), 2012, pp. 231-234.

[7] C. A. Chin, G. V. Crosby, T. Ghosh and R. Murimi, "Advances and Challenges of Wireless Body Area Networks for Healthcare Applications,” in Computing, Networking and Communications (ICNC), International Conference on, 2012, pp. 99-103.

[8] C.-H. Kang, S.-J. Wu and J.-H. Tarng, "A Novel Folded UWB Antenna for Wireless Body Area Network," Antennas and Propagation, IEEE Transactions on, Vol. 60, 2012, pp. 1139-1142. doi:10.1109/TAP.2011.2173101

[9] T. Bonnici, C. Orphanidou, D. Vallance, A. Darrell and L. Tarassenko, "Testing of Wearable Monitors in a Real-world Hospital Environment: What Lessons can be Learnt?” in Wearable and Implantable Body Sensor Networks (BSN), Ninth International Conference on, 2012, pp. 79-84.

[10] W. B. Xu, D. H. Wang, K. Niu, Z. Q. He and J. R. Lin, "A Channel Estimation Method Based on Distributed Compressed Sensing and Time-domain Kalman Filtering in OFDM Systems," in Broadband Network and Multimedia Technology (IC-BNMT), 4th IEEE International Conference on, 2011, pp. 157-161.

[11] S. Atapattu, C. Tellambura and H. Jiang, "Performance of an Energy Detector over Channels with Both Multipath Fading and Shadowing," Wireless Communications, IEEE Transactions on, Vol. 9, No. 12, 2010, pp. 3662-3670.doi:10.1109/TWC.2010.100110.091042

[12] H. Su and X. Zhang, "Battery-dynamics Driven Tdma mac Protocols for Wireless Body-area Monitoring Networks in Healthcare Applications," Selected Areas in Communications, IEEE Journal on, Vol. 27, No. 4, 2009, pp. 424-434. doi:10.1109/JSAC.2009.090507

[13] M. Balouchestani, K. Raahemifar and S. Krishnan, "Robust Wireless Sensors with Comoressed Sensing Theory," Communication in Computer and Information Science Journal of Springer, Vol. 293, 2012, pp. 608-619. doi:10.1007/978-3-642-30507-8_50

[14] P. K. Baheti and H. Garudadri, "An Ultra Low Power Pulse Oximeter Sensor Based on Compressed Sensing," in Wearable and Implantable Body Sensor Networks, BSN 2009. Sixth International Workshop on, 2009, pp. 144-148.

[15] M. Balouchestani, K. Raahemifar and S. Krishnan, "Wireless Body Area Networks with Compressed Sensing Theory," in Complex Medical Engineering (CME), ICME International Conference on, 2012, pp. 364-369.

[16] H. Kim, R. F. Yazicioglu, P. Merken, C. Van Hoof and Hoi-Jun Yoo, "ECG Signal Compression and Classification Algorithm With Quad Level Vector for ECG Holter System," Information Technology in Biomedicine, IEEE Transactions on, Vol. 14, 2010, pp. 93-100.

[17] S. M. Jadhav, S. L. Nalbalwar and A. Ghatol, “Artificial Neural Network Based Cardiac Arrhythmia Classification Using ECG Signal Data," Electronics and Information Engineering (ICEIE), International Conference on, 2010, pp. V1-228-V1-231.

[18] H. Mamaghanian, N. Khaled, D. Atienza and P. Vandergheynst, "Compressed Sensing for Real-Time Energy-Efficient ECG Compression on Wireless Body Sensor Nodes,” Biomedical Engineering, IEEE Transactions on, Vol. 58, No. 9, 2011, pp. 2456-2466. doi:10.1109/TBME.2011.2156795

[19] L. F. Polania, R. E. Carrillo, M. Blanco-Velasco and K. E. Barner, "Compressed Sensing Based Method for ECG Compression," in Acoustics, Speech and Signal Processing (ICASSP), IEEE International Conference on, 2011, pp. 761-764.

[20] W. Dullaert, H. Rogier, L. De Camillis and T. Dhaene, "Improving Link Quality of UWB Communication Links by Means of PSWF-basis Persuit Denoising," in Antennas and Propagation in Wireless Communications (APWC), IEEE-APS Topical Conference on, 2011, pp. 126-129.

[21] K. Kanoun, H. Mamaghanian, N. Khaled and D. Atienza, "A Real-time Compressed Sensing-based Personal Electrocardiogram Monitoring System,” in Design, Automation \& Test in Europe Conference \& Exhibition (DATE), 2011, pp. 1-6.

[22] A. S. Alvarado, C. Lakshminarayan and J. C. Principe, "Time-Based Compression and Classification of Heartbeats,” Biomedical Engineering, IEEE Transactions on, Vol. 59, 2012, pp. 1641-1648. 


\section{doi:10.1109/TBME.2012.2191407}

[23] A. M. R. Dixon, E. G. Allstot, A. Y. Chen, D. Gangopadhyay and D. J. Allstot, "Compressed Sensing Reconstruction: Comparative Study with Applications to ECG Bio-signals," in Circuits and Systems (ISCAS), 2011 IEEE International Symposium on, 2011, pp. 805-808.

[24] L. F. Polania, R. E. Carrillo, M. Blanco-Velasco and K. E. Barner, "Compressive Sensing for ECG Signals in the Presence of Electromyographic Noise," in Bioengineering Conference (NEBEC), 38th Annual Northeast, 2012, pp. 295-296.

[25] H. Mamaghanian, N. Khaled, D. Atienza and P. Vandergheynst, "Compressed Sensing for Real-Time Energy-Efficient ECG Compression on Wireless Body Sensor Nodes,” Biomedical Engineering, IEEE Transactions on,
Vol. 58, 2011, pp. 2456-2466.

doi:10.1109/TBME.2011.2156795

[26] P. Karthikeyan, M. Murugappan and S. Yaacob, "ECG Signals Based Mental Stress Assessment Using Wavelet Transform," in Control System, Computing and Engineering (ICCSCE), IEEE International Conference on, 2011, pp. 258-262.

[27] A. Chacko and S. Ari, "Denoising of ECG Signals Using Empirical Mode Decomposition Based Technique,” in Advances in Engineering, Science and Management (ICAESM), International Conference on, 2012, pp. 6-9.

[28] C. Ye, B. V. K. Vijaya Kumar and M. T. Coimbra, "Heartbeat Classification Using Morphological and Dynamic Features of ECG Signals,” Biomedical Engineering, IEEE Transactions on, Vol. 59, 2012, pp. 2930-2941. 